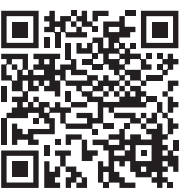

Palabras clave: Simulación clínica, formación docente, satisfacción

de estudiantes, facilitación.

Keywords: Clinical simulation, teacher training, student satisfaction, facilitation.

* Enfermera,

Profesor asistente.

* Enfermera, Profesor

instructor.

§ Enfermera Matrona,

Profesor asistente,

Coordinadora Nacional.

Unidad de Simulación Clínica de la

Universidad Santo

Tomás (UST). Chile.

Recibido: 11/09/2020

Aceptado: 30/11/2020

doi: $10.35366 / 97902$

\title{
Relación entre formación docente en metodología de simulación clínica y satisfacción usuaria en estudiantes de pregrado de carreras de salud
}

\author{
Relationship between teacher training in clinical \\ simulation methodology and user satisfaction in \\ undergraduate students of health careers \\ Claudia Palma-Guerra, * María José Cifuentes-Leal, ${ }^{\ddagger}$ \\ Paulina Espoz-Lara, ${ }^{\S}$ Cynthia Vega-Retamal, ${ }^{*}$ María Dolores Jaramillo-Larson ${ }^{\ddagger}$
}

\section{RESUMEN}

Implementar la simulación clínica (SC) en la Universidad Santo Tomás ha sido un desafío que involucró diversas áreas, como infraestructura, equipamiento, recursos humanos, así como docencia y capacitación, con la finalidad de instaurar de manera integral esta metodología para que docentes y estudiantes utilicen este nuevo modelo para el aprendizaje. La necesidad de capacitación docente para lograrlo motivó la realización de este estudio, cuyo objetivo es describir la relación entre formación docente en SC y satisfacción de estudiantes de pregrado de la Facultad de Salud. Se utilizó metodología descriptiva y retrospectiva, y se aplicó el "cuestionario prácticas docentes versión estudiante” de Reese C. y Jeffries P., a dos grupos, uno con talleres con docentes no capacitados y otro con docentes capacitados, con una muestra total de 1,895 estudiantes. Como resultado, se observa que ambos grupos de estudiantes se encuentran satisfechos con la SC, lo cual demuestra que existe la percepción de beneficio directo en el aprendizaje. Además, destaca la satisfacción de los estudiantes del grupo que cursó talleres con docentes capacitados, específicamente en el ítem de facilitación. Esto resulta importante para la toma de decisiones institucionales en miras de la mejora continua, para formar más docentes, entregándoles herramientas que permitan potenciar la implementación de la simulación clínica.

\section{ABSTRACT}

The implementation of the clinical simulation (CS) in Santo Tomas University has been a challenge that involved various areas like infrastructure, equipment, human resources and also teaching and training with the purpose of setting up this methodology as a whole so that professors and students are able to use this new learning model. The necessity of teaching the professors to meet this goal motivated this study, its main objective is to describe the relationship between professors trained in CS and undergraduate students'satisfaction in the Health Faculty. With the use of descriptive and retrospective methodology, applying the "teaching practices questionnaire student version" by Reese C. and Jeffries P. in two groups one with study workshops with professors without the training and the other one with trained professors, with a total of 1,895 students. As a result, we observed that the students in both groups are satisfied with the CS, that goes to show the perception of the direct benefit in learning. Stands out the satisfaction of students in the group that had their study workshop with trained professors, specifically in the facilitation item. This becomes important for the decision making in the institution looking for the continuous improvement, to form more professors giving them the tools that permit the potential in the implementation of clinical simulation.

\section{INTRODUCCIÓN}

T a simulación clínica (SC) se impone en el $ـ$ mundo como una de las principales y más efectivas herramientas metodológicas para la edu- cación en carreras del área de la salud. Constituye un método de enseñanza y aprendizaje efectivo para lograr en los estudiantes el desarrollo de un conjunto de competencias necesarias que posibiliten alcanzar los objetivos del perfil del

Citar como: Palma-Guerra C, Cifuentes-Leal MJ, Espoz-Lara P, Vega-Retamal C, Jaramillo-Larson MD. Relación entre formación docente en metodología de simulación clínica y satisfacción usuaria en estudiantes de pregrado de carreras de salud. Simulación Clínica. 2020; 2 (3): 133-139. https://dx.doi.org/10.35366/97902 
egresado. Tiene el propósito de ofrecer al estudiante la oportunidad de realizar una práctica similar a la que realizará en su interacción con la realidad en las diferentes áreas o escenarios docente-asistenciales. ${ }^{1}$

A nivel internacional, las escuelas de salud están invirtiendo en tecnología para asegurar que sus centros de aprendizaje y de simulación sean lugares en donde los estudiantes pueden participar en simulaciones clínicas verídicas y libres de riesgo, que los prepararán lo mejor posible para el mundo real en el cual ejercerán. ${ }^{1}$

La simulación clínica cobra importancia al ser utilizada como una herramienta para facilitar el aprendizaje, como por ejemplo, en los estudiantes de enfermería, quienes son sometidos a una experiencia en la que se logra realizar una práctica análoga a la realidad asistencial, en la cual interaccionan conocimientos, habilidades y factores humanos que proveen a los estudiantes de un método de aprendizaje, entrenamiento sistemático y repetido de habilidades prácticas, que posteriormente permitirán desarrollar un conjunto de destrezas y competencias, en un ambiente donde el error está permitido y no se encuentre en riesgo la vida de los usuarios. ${ }^{2}$

En la Universidad Santo Tomás (UST), la implementación de la metodología de simulación clínica nace como un proyecto en el año 2014, momento en el cual se elabora la fundamentación y el plan de trabajo. Luego, en el año 2016 se construye el primer centro piloto en sede La Serena. Producto de su evaluación funcional en cuanto a infraestructura y equipamiento, en el año 2017 se construye un segundo centro con las mejoras definidas, en sede Concepción. Después, entre los años 2018 y 2019, se concreta la construcción e implementación de 11 centros de simulación clínica en el resto de las sedes de la institución, alcanzando una cobertura total de 13 centros en la UST a nivel nacional. En la actualidad, los centros de simulación benefician a un total de 14,000 estudiantes de las carreras de: Enfermería, Fonoaudiología, Kinesiología, Nutrición y Dietética, Tecnología Médica y Terapia Ocupacional. ${ }^{3}$

El perfil de los estudiantes de la UST se caracteriza por jóvenes, que en su mayoría, son la primera generación de su familia en acceder a la educación superior y que mayoritariamente trabaja para costear parte de sus estudios. ${ }^{4}$

El modelo educativo de la institución considera a los estudiantes como el centro y protagonista del aprendizaje, lo cual es coherente con la metodología de la SC, que pone énfasis en el desarrollo autónomo de los alumnos como gestor de su aprendizaje, cambiando el paradigma conductista de la educación superior hacia un paradigma constructivista. ${ }^{5}$ Este proceso se ve facilitado por los docentes que conforman el cuerpo académico de la institución.

La SC muestra resultados positivos en pregrado; sin embargo, el cambio de paradigma educativo implica mayor responsabilidad del estudiante, lo que podría generar una valoración negativa. ${ }^{6}$ Considerando lo expuesto anteriormente, dentro del proyecto general de este estudio se encuentra la hipótesis de que los estudiantes tengan una percepción negativa frente a la implementación de la simulación clínica. Por tanto, es importante para la Facultad de Salud observar este fenómeno e implementar de manera oportuna estrategias que puedan significar un aumento en la motivación de los estudiantes frente a su aprendizaje, lograr un mejor aprovechamiento de la metodología implementada y, como consecuencia de ello, un mejor desempeño en las actividades prácticas.

\section{SATISFACCIÓN DE LOS ESTUDIANTES CON LA METODOLOGÍA}

De acuerdo con la revisión de diferentes autores, la satisfacción del estudiante con la simulación clínica, evidencia la eficiencia de los sistemas educativos y administrativos, ya que son ellos los principales usuarios que pueden valorar los servicios de las universidades, puesto que al ser los protagonistas de este proceso educativo, emiten sus opiniones que reflejan sus percepciones, las que sirven como indicador de la calidad de enseñanza, del desarrollo de los programas académicos y del mejoramiento de la gestión. Al tener a un estudiante satisfecho se confirma la calidad de la educación brindada, lo que a su vez generará un profesional humanizado y preparado. ${ }^{7}$

Dentro de los diferentes estudios relacionados con la satisfacción de los estudiantes con la metodología, en general, se ha encontrado que la experiencia mejoró su aprendizaje y sus habilidades para tomar decisiones. Los encuestados emitieron respuestas muy positivas con respecto a la transferibilidad, el realismo y el valor de la experiencia de simulación con el paciente. ${ }^{6}$

Esta metodología reporta mayor satisfacción personal con la experiencia simulada, al integrar la teoría con la práctica; permitiendo el desarrollo del pensamiento crítico al priorizar acciones, el desarrollo de destrezas, seguridad clínica, la 
comunicación, el trabajo en equipo y la ética profesional. $^{8}$

El modelo pedagógico que subyace en estas formas de intervención educativa se sustenta en una concepción de aprendizaje centrada en la actividad del alumno y en su construcción cognitiva que resulta de su reflexión y análisis, así como en una intervención por parte del docente, que orienta y facilita los aprendizajes en el alumno. ${ }^{9}$ Es importante destacar lo anterior, ya que propone un cambio en la posición del estudiante, desde un rol pasivo a uno completamente activo.

\section{CAPACITACIÓN DOCENTE EN SIMULACIÓN CLÍNICA}

Los instructores en SC requieren de entrenamientos y habilidades para incorporar la simulación en sus actividades de enseñanza. Una simulación efectiva requiere de instructores que tengan habilidades de enseñanza centrada en aprendizaje por medio de la facilitación, haciendo uso de escenarios de simulación. ${ }^{10}$

La capacitación es la que le permitirá conocer las reglas de la simulación que estimulen la responsabilidad, la autodirección y la motivación de los estudiantes. También deberá incluir los mecanismos que permitan a los estudiantes poder cometer errores, mantener la salvedad o seguridad, crear un ambiente no competitivo y tener claridad de los roles de los participantes. ${ }^{10}$

$\mathrm{El}$ rol docente en SC exige que éste migre desde un rol protagónico y demostrativo a uno de facilitador y entrenador, siendo actor secundario, guiando a los estudiantes para que construyan en conjunto su aprendizaje. ${ }^{10}$

Para la implementación de esta metodología, los instructores deben estar debidamente capacitados con uso de un lenguaje común y desarrollo estandarizado de las prácticas pedagógicas que evidencien un proceso armónico en todas las simulaciones para evitar que el estudiante tenga incertidumbre en su aprendizaje y que se sienta satisfecho con lo que recibe. Lo anterior, además, repercutirá en la calidad de la formación profesional. ${ }^{10}$

Según Amaya $\mathrm{A}^{11}$ para llevar a cabo la simulación clínica es necesario contar con docentes capacitados que posean un perfil académico, claridad y adaptación conceptual, las cuales estén cimentadas en una amplia experiencia clínica. Lo anterior, logrará que el docente pueda contextualizar los distintos roles que los estudiantes han de realizar a la hora de enfrentar el escenario clínico.
Reforzando la idea anterior, el docente asume el papel de mediador en simulación clínica, que resulta importante a la hora de promover aprendizajes significativos, especialmente en la etapa de debriefing, donde se requiere de gran creatividad para moderar la discusión y fomentar la autoevaluación, mediante el desarrollo de habilidades descriptivas, orientando al conocimiento. ${ }^{12}$

Es por lo anterior, que la capacitación docente para la UST se impuso como una necesidad y desafío, no sólo desde el punto de vista del cambio de posición del docente instructor al rol facilitador, sino que además se hace imprescindible garantizar la homogeneidad de los procesos en los 13 centros con los que cuenta la institución a lo largo del país, considerando que un gran número de docentes y estudiantes se verán beneficiados con la implementación de la metodología.

Inicialmente, en el proceso de implementación de esta metodología en la UST se realizaron capacitaciones presenciales a docentes en las sedes piloto y prototipo, entre los años 2016 y 2018. Paralelo a ello se capacitó de manera formal a quienes asumirían el cargo de coordinadores de los centros de simulación clínica, con diplomados en universidades con experiencia en el área. En el año 2019 se dictó el curso de "herramientas de simulación clínica" de 30 horas, en modalidad E-learning, cuyo objetivo era establecer las bases teóricas de la metodología. En diciembre del mismo año se finalizó el programa con un total de 1,909 docentes capacitados mediante este curso. ${ }^{3}$

Al considerar que los planes de estudio de las seis carreras de la Facultad de Salud que utilizan la metodología en simulación ya iniciaron sus actividades docentes en el año 2018, y que las cohortes de estas carreras en el año 2019 proyectaban alrededor de $2 \%$ de sus horas de asignaturas con esta metodología (salvo Enfermería que tiene $7 \%$ de las horas de su plan de estudio utilizando esta estrategia metodológica), se planteó la necesidad de contar con resultados de satisfacción por parte de los estudiantes en cuanto a la implementación de esta metodología, en miras de la mejora continua del trabajo realizado y para dar respuesta a las necesidades de los docentes en cuanto a capacitación.

El siguiente estudio busca establecer si existe relación entre la formación docente en simulación clínica y la satisfacción del estudiante de pregrado en el uso de esta metodología didáctica.

Los resultados de este estudio permitirán a la institución atender las necesidades de los estudiantes en cuanto al aprendizaje bajo esta 
metodología activa, tomar decisiones en relación con las necesidades de capacitación de los académicos y transferir algunas de sus ventajas a otras áreas del quehacer docente.

\section{MATERIAL Y MÉTODOS}

Se trata de un estudio de diseño descriptivo retrospectivo. La recolección de datos se realizó con el instrumento "Cuestionario de prácticas docentes" de Jeffries P. del año 2012, en su versión traducida al español, siendo éste un extracto de su versión oficial en inglés y validado por de Reese C. y Jeffries P. ${ }^{13}$ Este cuestionario considera elementos diseñados para examinar las actitudes hacia el aprendizaje activo, elementos de colaboración, diversas formas de aprendizaje y altas expectativas. ${ }^{14}$

Dicho instrumento cuenta con 18 preguntas con respuestas tipo Likert que fue aplicado a 1,895 estudiantes de la Facultad de Salud de la UST en 13 sedes a nivel nacional, durante el segundo semestre del año 2018 y primer semestre del año 2019.

Los estudiantes a quienes se les aplicó el cuestionario corresponden a las carreras de Enfermería, Kinesiología, Nutrición y Dietética y Fonoaudiología. Al finalizar los talleres de simulación programados durante el semestre, los alumnos respondieron el instrumento de manera voluntaria y anónima.

El análisis estadístico se realizó en SPSS versión 19, se trabajó con tablas de frecuencia, distribución y prueba relación $\left(\chi^{2}\right)$.

Para lo anterior, se distribuyeron dos grupos de encuestados: estudiantes que experimentaron talleres de SC con docentes no capacitados versus

Tabla 1: Listado de preguntas seleccionadas del
cuestionario de prácticas docentes.

Cuestionario prácticas docentes (B) (versión estudiante) de Reese C. y Jeffries P. (2012). estudiantes que ejecutaron talleres con docentes capacitados. Se consideró al "docente no capacitado" (DNC) aquel que sólo había recibido formación en talleres y cursos presenciales teóricos entre los años 2016 y 2018 con un promedio de 40 horas de formación. El "docente capacitado" (DC) es aquel que además de recibir los talleres presenciales ya mencionados, participó en el curso "Herramientas de SC" en modalidad E-learning y que suma horas de experiencia en SC como instructor, ejecutando talleres durante el año 2018 y primer semestre del 2019 consideradas "horas de vuelo".

Para realizar el análisis se seleccionaron del cuestionario cinco preguntas que son las que se relacionan directamente con el objetivo de este estudio. En la Tabla 1 se especifican las preguntas elegidas.

Las respuestas a las preguntas del cuestionario fueron asociadas en dos grandes grupos: "de acuerdo" $y$ "desacuerdo", con lo cual se realizó el análisis estadístico mencionado. Se consideró $p$ > 0.05 , concluyendo que no existe relación estadísticamente significativa entre los grupos DNC y DC.

\section{RESULTADOS}

Respecto a la satisfacción de estudiantes en los criterios seleccionados, al relacionar las variables docente no capacitado (DNC) y docente capacitado (DC), tal como se muestra en la tabla 2, es posible observar que en las preguntas número 1 , 5, 14 y 18 al ser "p-valor" mayor a 0.05 no se rechaza la hipótesis planteada en la investigación, lo que en definitiva, indica que no existe relación significativa respecto a la satisfacción de los estudiantes encuestados considerando a aquellos que participaron de simulación con docentes no capacitados (DNC) y docentes capacitados (DC). Los resultados generales evidencian el alto grado de satisfacción de los estudiantes con la SC independiente del nivel de formación de los docentes en la metodología.

No obstante, en la pregunta relacionada con la satisfacción "los objetivos de la simulación fueron claros y fáciles de comprender", p-valor resultó ser de 0.003 , por lo tanto se rechaza la hipótesis, esto indica que sí hay relación en esa pregunta y que por ende existen diferencias entre la facilitación de los docentes capacitados versus los no capacitados.

\section{DISCUSIÓN}

De acuerdo con los resultados obtenidos, es posible observar que la capacitación docente, 
Tabla 2: Resultados del análisis estadístico del cuestionario de prácticas docentes.

\begin{tabular}{|c|c|c|c|c|c|}
\hline \multirow[b]{2}{*}{ Preguntas } & \multicolumn{2}{|c|}{$\begin{array}{l}\mathrm{DNC}(\mathrm{n}=1,395) \\
\mathrm{n}(\%)\end{array}$} & \multicolumn{2}{|c|}{$\begin{array}{l}\mathrm{DC}(\mathrm{n}=500) \\
\mathrm{n}(\%)\end{array}$} & \multirow[b]{2}{*}{$\mathrm{p}$} \\
\hline & Desacuerdo & De acuerdo & Desacuerdo & De acuerdo & \\
\hline $\begin{array}{l}\text { 1: "He tenido la oportunidad durante la simulación para } \\
\text { analizar las ideas y conceptos que se enseñan” }\end{array}$ & $104(7.5)$ & 1,291 (92.5) & $26(5.2)$ & 474 (94.8) & 0.08 \\
\hline $\begin{array}{l}\text { 5: "Aprendí de las observaciones formuladas por el profesor } \\
\text { antes, durante o después de la simulación” }\end{array}$ & $93(6.6)$ & 1,300 (93.2) & $30(6.0)$ & $470(94.8)$ & 0.6 \\
\hline $\begin{array}{l}\text { 12: "Los objetivos de la simulación fueron claros y fáciles } \\
\text { de comprender" }\end{array}$ & 116 (8.31) & $1,277(91.54)$ & $21(4.2)$ & 475 (95) & 0.003 \\
\hline $\begin{array}{l}\text { 14: "El instructor fue capaz de responder a las necesidades } \\
\text { individuales de los alumnos durante la simulación" }\end{array}$ & $88(6.32)$ & 1,297 (92.98) & $29(5.8)$ & $463(90.6)$ & 0.7 \\
\hline $\begin{array}{l}\text { 18: "El uso de actividades de simulación hacen que mi } \\
\text { tiempo de estudio sea más productivo" }\end{array}$ & $141(10.1)$ & 1,250 (89.6) & 416 (89.5) & $49(10.5)$ & 0.8 \\
\hline
\end{tabular}

Cuestionario prácticas docentes (B) (versión estudiante) de Reese C. y Jeffries P. (2012).

a modo general, no tiene vínculo significativo con la satisfacción de los estudiantes. Esto puede deberse a que la metodología en sí es atractiva y brinda resultados positivos evidentes. Otros factores influyentes serían, por ejemplo, que los estudiantes son novatos en la metodología y que la infraestructura, tecnología y equipamiento les podría resultar novedosos. Es posible afirmar que los alumnos pertenecientes a la Facultad de Salud de la UST se encuentran satisfechos con la implementación de la metodología en diferentes áreas, independiente del nivel de capacitación del docente. Ello quedó demostrado en el análisis de las preguntas número 1, 5, 14 y 18.

En otros estudios que apoyan esta satisfacción general de los estudiantes con la simulación clínica, se establece que existe una importante conexión entre la satisfacción y la motivación para aprender, conexión que es de particular interés en la generación actual de estudiantes, inundados de los más diversos estímulos. Así, un estudiante motivado aprende más y mejor, creyendo en la potencial utilidad de lo que aprende para su práctica futura. ${ }^{15}$

Según MacKinnon y colaboradores, dentro de los aspectos que los estudiantes valoran como agentes que brindan mayor seguridad para enfrentarse a experiencias clínicas reales, se encuentra la simulación clínica porque brinda entrenamiento en habilidades de razonamiento crítico, una mejor integración de los contenidos de clases teóricas y enseñanzas clínicas, toma de decisiones, y que es una herramienta pragmática para contextualizar y lograr conocimientos. ${ }^{16}$

Específicamente, en la pregunta número 12, los alumnos mostraron una mayor satisfacción en el grupo de los docentes capacitados; por lo tanto, se concluye que la formación docente sí influye en el área de la facilitación en simulación clínica. Esto último se apoya al considerar que los docentes en muchas ocasiones vienen del mundo sanitario y carecen de formación docente, o bien, reciben capacitación de forma incipiente. Se resalta la necesidad de capacitar en específico a los educadores en esta metodología, ya que la docencia con simulación resulta efectiva cuando se realiza bajo las condiciones adecuadas y se ha demostrado que los estudiantes muestran mayores niveles de satisfacción en ambientes seguros, simulados y similares a la realidad. ${ }^{17}$

A menudo se confunde el enseñar con un simulador con la metodología de la simulación. Es por ello que se hace necesario identificar las prácticas docentes que se asocian a los mejores resultados de aprendizaje y a formar a los educadores en la mejor evidencia docente disponible. ${ }^{18}$ Con relación a lo anterior, es preciso, al tener en cuenta los resultados de este estudio, enfocar la capacitación de los docentes en herramientas para fortalecer en ellos las habilidades de facilitación, además de identificar, a través del acompañamiento docente, otras áreas necesarias de desarrollar, con el objetivo de mejorar los resultados del aprendizaje de los estudiantes. 
Esto es posible abordar a través de cápsulas de formación en simulación clínica con aspectos teóricos y prácticos y acompañamiento docente presencial en los primeros talleres, entregando feedback personalizado respecto a lo observado.

Es por ello que, tras este estudio, se establece un plan de trabajo que considera talleres de capacitación para todos los docentes que participan en actividades de simulación clínica como responsables de asignaturas. El programa de capacitación declarado en simulación clínica para docentes de la institución se detalla en la Tabla 3.

En relación al curso que se dicta en modalidad E-learning, la primera versión, contó con la participación de 262 docentes y la segunda con 219 docentes. Esto representa 25\% del total de docentes de la Facultad de Salud a nivel nacional (1,935 docentes registrados, según datos del sistema académico institucional, CLAS en abril de 2019).

Los docentes que han asistido a los talleres de generación de material académico y debriefing son 291 en total para el segundo semestre del año 2019 (no es posible expresar en porcentaje, ya que no se cuenta con el dato oficial de docentes para ese periodo).

Para concluir, es importante garantizar el acompañamiento y la facilitación del docente durante la simulación clínica para la correcta adquisición de habilidades, lo cual disminuye el error en el contexto real y ejerce en los estudiantes un efecto directo en la motivación que permite obtener mejores resultados en ellos. ${ }^{19}$ Apoyando lo anterior, es necesario asumir procesos formativos en la institución, liderados por personas interesadas en pedagogías innovadoras

\begin{tabular}{|c|c|c|}
\hline \multicolumn{3}{|c|}{$\begin{array}{l}\text { Tabla 3: Programa de capacitación en simulación } \\
\text { clínica para docentes de la UST. }\end{array}$} \\
\hline $\mathrm{N}^{0}$ & Título del taller o curso & Horas de capacitación \\
\hline 1 & $\begin{array}{l}\text { Curso de “Herramientas en simulación clínica” } \\
\text { versión E-learning }\end{array}$ & 30 \\
\hline 2 & $\begin{array}{l}\text { Taller de "Generación de material académico de } \\
\text { simulación clínica” }\end{array}$ & 8 \\
\hline 3 & $\begin{array}{l}\text { Taller de "Debriefing: elemento clave de la } \\
\text { simulación clínica” }\end{array}$ & 8 \\
\hline 4 & $\begin{array}{l}\text { Programa de acompañamiento docente presencial } \\
\text { individual }\end{array}$ & Según necesidad* \\
\hline
\end{tabular}

con la finalidad de apoyar al cuerpo académico en mejorar sus experiencias docentes. ${ }^{20}$ Según esto, sería ideal avanzar en la transversalidad del docente "facilitador" no sólo en SC, sino en todo el proceso enseñanza-aprendizaje. Esto sería posible generando instancias de entrenamiento con estrategias didácticas, logrando coherencia de facilitación en todo el proceso formativo, desde las clases teóricas hasta la experiencia clínica, promoviendo en los estudiantes una mayor participación y responsabilidad en la gestión de su aprendizaje.

Respecto al plan de capacitación en simulación clínica de la UST para el año 2020, éste consideraba mantener la estructura inicial, avanzando hacia temas como el rol del paciente simulado y evaluación en simulación clínica. Dado el contexto mundial del inicio de este año, se nos plantea la necesidad de redefinir las áreas que representan la mayor necesidad de capacitación docente para construir un programa 2020-2021 dirigido en fortalecer la facilitación en escenarios virtuales, en los cuales se desarrolla la simulación clínica hoy y en donde se ha centrado el quehacer de la unidad de simulación clínica.

\section{REFERENCIAS}

1. Utili F. Simulación en el aprendizaje, práctica y certificación de las competencias en medicina. ARS Med [Internet]. 2007 [citado 14 de may 2019]; 36 (2): 152-163. Disponible en: https://doi.org/10.11565/ arsmed.v36i2.154

2. Juguera L, Díaz J, Pérez M, Leal C, Rojo A, Echeverría P. La simulación clínica como herramienta pedagógica. Percepción de los alumnos de grado en Enfermería en la Universidad Católica San Antonio de Murcia. Enferm Glob [internet]. 2014 [citado 14 de may 2019]; 13 (33): 175-190. Disponible en: http://scielo.isciii.es/ pdf/eg/v13n33/docencia3.pdf

3. Espoz Lara P. Informe de gestión unidad de Simulación clínica UST, primer semestre 2019. Facultad de Salud, Chile: Universidad Santo Tomás; 2019.

4. Universidad Santo Tomás: Dirección académica UST Santiago. Resultados encuesta de caracterización realizada a estudiantes de UST Santiago; 2019.

5. Alarcón Rojas G. Plan de desarrollo. Facultad de Salud, Chile: Universidad Santo Tomás; 2019.

6. Abdo A, Ravert P. Student Satisfaction with Simulation Experiences. Clin Simul Nurs [Internet]. 2006 [citado 30 de may 2019]; 2 (1): 13-16. Disponible en: https:// doi.org/10.1016/j.ecns.2009.05.009

7. Campos M, Campos S, González R, Romero N. Satisfacción con la simulación clínica de alta fidelidad en estudiantes de Enfermería [Tesis]. Chile: Universidad del Bío-Bío; 2015. [Citado 16 de jun 2019]. Disponible en: http://repobib.ubiobio.cl/ jspui/bitstream/123456789/2636/1/Campos\%20 Rojas\%2C\%20Silvana\%20Natalia.pdf 
8. Castillo-Arcos L, Maas-Góngora L. Percepción de satisfacción de los estudiantes de enfermería en el uso de la Simulación Clínica. Ra Ximhai [Internet]. 2017 [citado 16 de may 2019]; 13 (2): 63-76. Disponible en: http://www.revistas.unam.mx/index.php/rxm/article/ view/71499/63092

9. Piña-Jiménez I, Amador-Aguilar R. La enseñanza de la enfermería con simuladores, consideraciones teórico-pedagógicas para perfilar un modelo didáctico. Enfermería Universitaria [Internet]. 2018 [citado 27 de jun de 2019]; 12 (3). Disponible en: http:// www.revista-enfermeria.unam.mx/ojs/index.php/ enfermeriauniversitaria/article/view/135

10. Urra E, Sandoval S, Irribarren F. El desafío y futuro de la simulación como estrategia de enseñanza en enfermería. Investigación Educ Médica [Internet]. 2017 [citado 6 de jul 2019]; 6 (22): 119-125. Disponible en: http://www.scielo.org.mx/scielo.php?script=sci arttext\&pid $=$ S2007-50572017000200009\&lng $=e s$

11. Amaya A. Simulación clínica: ipretende la educación médica basada en la simulación remplazar la formación tradicional en medicina y otras ciencias de la salud en cuanto a la experiencia actual con los pacientes? Univ Méd Bogotá [Internet]. 2008 [citado 21 de jul 2019]; 49 (3): 399-405. Disponible en: https://www.redalyc.org/pdf/2310/231016421008.pdf

12. Ruda-Rodríguez N. Simulación clínica en la mediación pedagógica y su relación con la práctica clínica. ISUB [Internet]. 2014 [citado 21 de jul 2020]; 1 (2): 231 243. Disponible en: http://revistasdigitales.uniboyaca. edu.co/index.php/rs/article/view/125

13. Reese C. Effective teaching in clinical simulation: development of the student perception of effective teaching in clinical simulation scale [Doctoral thesis Internet]. Indiana University; 2009 [citado 6 de jul 2019]. Disponible en: https://core.ac.uk/download/ pdf/46956276.pdf

14. Zapko K, Ferranto M, Blasiman R, Shelestak D. Evaluating best educational practices, student satisfaction, and self-confidence in simulation: A descriptive study. Nurse Education Today [Internet]. 2018 [citado 6 de jul 2019]; 60: 28-34. Disponible en: https://doi.org/10.1016/j.nedt.2017.09.006
15. Baptista RCN, Martins JCA, Pereira MFCR, Mazzo A. Satisfação dos estudantes com as experiências clínicas simuladas: validação de escala de avaliação. Rev Lat Am Enfermagem [Internet]. 2014 [citado 6 de jul 2019]; 22 (5): 709-715. Disponible en: https://doi. org/10.1590/0104-1169.3295.2471

16. MacKinnon K, Marcellus L, Rivers J, Gordon C, Ryan M, Butcher D. Student and educator experiences of maternal-child simulation-based learning: a systematic review of qualitative evidence protocol. JBI Database System Rev Implement Rep. 2015; 13 (1): 14-26. doi: 10.11124/jbisrir-2015-1694.

17. Ferri P, Cremonini V, Artioli G, Sarli L, Piccioni E, Rubbi I. Nursing students' experiences of and satisfaction with the clinical learning enviroment: the role of educational models in the simulation laboratory and in clinical practice. Acta Biomed. 2015; 86 (3). Disponible en: https://iris.unimore.it/ handle/11380/1113279\#.X8FtlmgzY2w

18. Opazo E, Rojo E, Maestre J. Modalidades de formación de instructores en simulación clínica: el papel de una estancia o pasantía. Educación Médica [Internet]. 2017 [citado 10 de ago 2020]; 18 (1): 22-29. Disponible en: https://doi.org/10.1016/j.edumed.2016.07.008

19. Gamboa D, Pérez M, Martínez E. Percepción de los estudiantes de enfermería de la Pontificia Universidad Javeriana sobre el aprendizaje a través de la simulación clínica [tesis en internet]. Colombia: Pontificia Universidad Javeriana; 2013 [citado 20 de ago 2019]. Disponible en: http://hdl.handle.net/10554/13761

20. Niño CA, Vargas NG, Barragán JA. Fortalecimiento de la Simulación clínica como herramienta pedagógica en enfermería: experiencia de internado. Revista Cuidarte [internet]. 2015 [citado 20 de sep 2019]; 6 (1): 970975. Disponible en: http://dx.doi.org/10.15649/ cuidarte.v6i1.161

Correspondencia:

Claudia Palma Guerra

Av. Ramón Picarte 1160, 5090000, Valdivia, Chile.

Teléfono: + 569 7777-3427

E-mail: claudiapalma1@santotomas.cl 